\title{
Light emission properties of a discharge induced in a gas flow by terahertz waves in the vacuum and extreme ultraviolet range
}

\author{
S.V. Razin, A.V. Sidorov, A.G. Luchinin, D.S. Sidorov, S.V. Golubev, M.Yu. Glyavin, \\ A.V. Vodopyanov
}

Institute of Applied Physics of Russian academy of Sciences, Nizhny Novgorod, Russia, sevraz@appl.sci-nnov.ru

\section{Introduction}

Research activity on the discharge, sustained by powerful radiation of the terahertz frequency range, has been actively conducted at the Institute of Applied Physics for a number of years [1-4]. Such a discharge is a new object of the plasma physics, and its investigations are of both fundamental and applied interest. This paper presents the results of experimental studies of the terahertz discharge plasma emission in the VUV spectral range $(112-180 \mathrm{~nm})$ and EUV spectral range (12 - $17 \mathrm{~nm})$. The discharge was induced in a nonuniform gas flow ( $\mathrm{Ar}, \mathrm{Kr}, \mathrm{Xe}$ ) under the action of a focused beam of terahertz waves.

\section{Experimental Setups}

The experiments were carried out at two different facilities (see the block diagram in Fig. 1). The sources of terahertz radiation were gyrotrons. One gyrotron operated in a pulsed mode (setup № 1, frequency $0.67 \mathrm{THz}$, pulse duration $20 \mu \mathrm{s}$, power 40 $\mathrm{kW}$, intensity in the beam focus $16 \mathrm{MW} / \mathrm{cm}^{2}$ (for a detailed description of the setup, see [3 - 5])), another gyrotron operated in a CW mode (setup №2, frequency $0.263 \mathrm{THz}$, power $1 \mathrm{~kW}$, intensity in the beam focus $15 \mathrm{~kW} / \mathrm{cm}^{2}$ ).

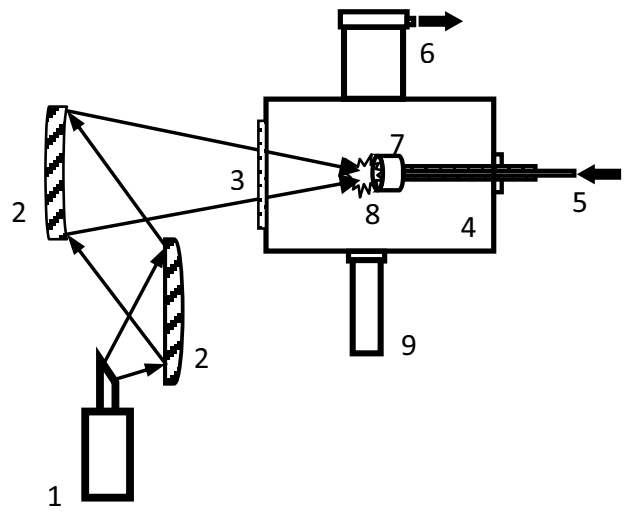

Fig. 1. Block diagram of experimental facilities. 1 gyrotron, 2 - quasi-optical beamline, $3-\mathrm{THz}$ vacuum window of the discharge chamber, 4 - discharge chamber, 5 - working gas supply system, 6 - vacuum pumping system, 7 - nonuniform gas flow formation unit, 8 discharge plasma, 9 - VUV photoelectron multiplier or EUV solid state detector with set of suitable filters.

The discharge was ignited in a nonuniform gas flow $(\mathrm{Ar}, \mathrm{Kr}, \mathrm{Xe})$ under the action of a focused beam of terahertz waves. The gas flow was formed by injecting gas into the discharge chamber through a small-diameter hole $(80 \mu \mathrm{m})$ under the pressure up to several atmospheres. An absolutely calibrated photoelectron multiplier (PMT) and a semiconductor detector in combination with a set of appropriate filters were used to measure the VUV (spectral range 112 - $180 \mathrm{~nm}$ ) and EUV (spectral range $12-17 \mathrm{~nm}$ ) emission of plasma.

\section{Experimental Results}

The performed experiments showed that the maximum power of the VUV and EUV radiation was observed in a point-like discharge with characteristic dimensions of about $1 \mathrm{~mm}[2$ - 4]. In Fig. 2 for an example, a photo of such a point-like discharge realized on the above-described setup №2 with a CW mode of the gyrotron operating is given. It should be noted that earlier at the setup №1 we have already performed measurements of the VUV emission power of the point-like discharge. In particular, in the spectral range $112-180 \mathrm{~nm}$ a value of the order of 10 $\mathrm{kW}$ was obtained in argon $[3,4]$.

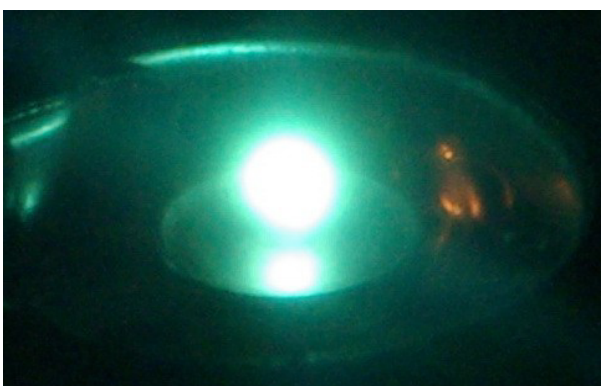

Fig. 2. A time-integrated photo of the emission of the pointlike discharge plasma in a nonuniform flow of xenon in the visible spectral range. Setup № 2, the background pressure in the discharge chamber is $4 \cdot 10^{-3}$ Torr, the terahertz radiation is propagated from top to bottom.

Analysis of the experimental data showed that the most promising in terms of generation of both vacuum and extreme ultraviolet is a point-like discharge in the xenon flow. Xenon multiply charged ions emit a lot of intense lines in the VUV and EUV spectral ranges [6], in particular, in the area of 13.5 $\mathrm{nm}$, which is of interest for high-resolution microlithography.

Fig. 3 shows the oscillogram of the VUV signal from the PMT obtained at setup №1 in the xenon flow. It is necessary to pay attention to the intense afterglow, the duration of which exceeds the duration of the terahertz pulse by several tens of times (it should be noted in the same way that an extremely long afterglow was observed earlier in other rare gases, in particular, in $\operatorname{Ar}[3,4])$. In addition, the intensity of the VUV radiation increased during the entire terahertz pulse. Hence it can be concluded that 
an increase in the gyrotron pulse duration should lead to a shift the emission spectrum of the discharge into the shorter-wave region. Probably, this should also lead to an increase in the intensity of the extreme ultraviolet.

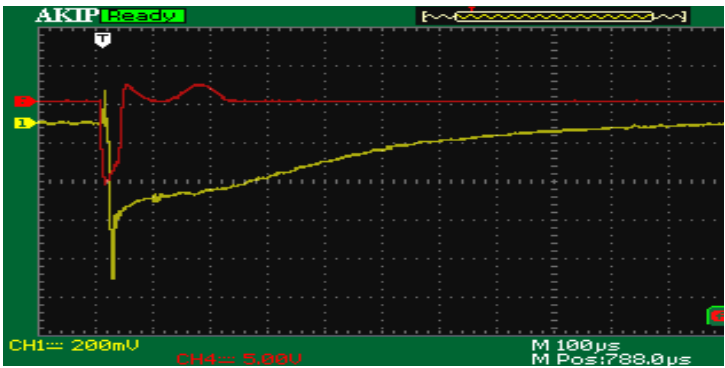

Fig. 3. Dynamics of the emission of a point-like discharge in the xenon flow in the VUV spectral region (the oscillogram of the signal from the PMT at a background pressure of $5 \cdot 10^{-3}$ Torr, lower waveform, setup №1). The upper waveform is a high-voltage pulse at the gyrotron cathode, the duration of which is approximately equal to the $\mathrm{THz}$ pulse duration.

According to experiments, the pressure of the gas injected into the discharge chamber has a significant effect on the plasma characteristics [5], and, consequently, on the generation of VUV and EUV emission. Fig. 4 shows the dependence of the VUV power of the plasma of a point-like discharge in the xenon flow on the pressure in the gas inlet system, obtained at setup № 2 at a maximum power of $\mathrm{THz}$ radiation of $1 \mathrm{~kW}$. It should be noted that at the same time the vacuum evacuation rate (up to $2000 \mathrm{l} / \mathrm{s}$ ) provided a low background pressure in the discharge chamber, and as a consequence, the maintenance of the point-like mode of the discharge. As can be seen from the Fig. 4, the power of the vacuum ultraviolet emitted by the plasma increases with increasing gas pressure in the gas-supply system.

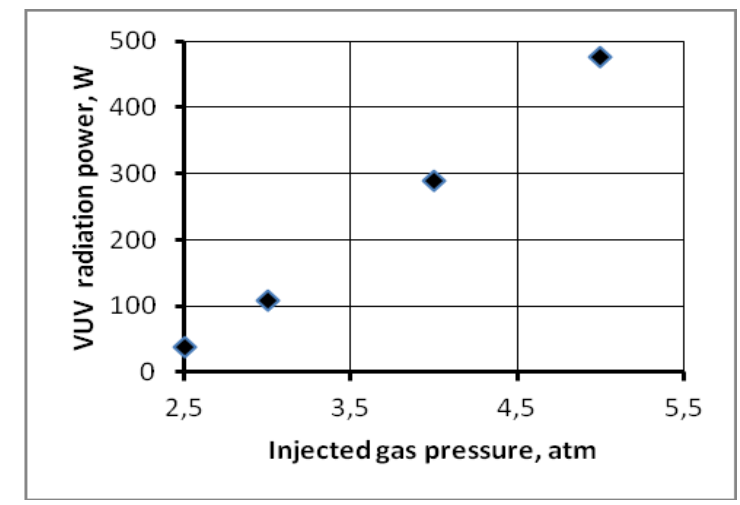

Fig. 4. The dependence of the VUV power emitted by the plasma of a point-like discharge in the xenon flow on the pressure in the gas-supply system (setup №2, power of terahertz radiation is $1 \mathrm{~kW}$ ).

As for the EUV radiation of the $\mathrm{THz}$ discharges described above, the analysis of the signals from the semiconductor detector showed that specific power of the EUV radiation of the plasma in the wavelength range $12-17 \mathrm{~nm}$ is $0.1 \mathrm{~W} / \mathrm{mm}^{3}$ for set up №1, and 0.01 $\mathrm{W} / \mathrm{mm}^{3}$ for set up №2.

\section{Conclusion}

Thus, the performed experiments showed that the discharge plasma created by focused terahertz radiation in a nonuniform flow of rare gases emits both a vacuum (112 - $180 \mathrm{~nm})$ ultraviolet and extreme ultraviolet $(12-17 \mathrm{~nm})$. In the conditions described above the power of the VUV radiation is large and reaches several tens of percent of the $\mathrm{THz}$ power supplied to the discharge. At the same time, the emitted EUV power is only a portion of a percent of the gyrotron radiation power. In the case of setup №1, an increase in the degree of conversion of $\mathrm{THz}$ radiation to an extreme ultraviolet is possible, apparently, by increasing the duration of the terahertz pulse of the gyrotron. Under the conditions of operation of the setup №2, an increase in the conversion degree is possible by increasing the $\mathrm{THz}$ radiation power and the pressure in the gas-supply system (in this case, it is necessary to maintain the point-like mode of the discharge, i.e., to keep the background pressure in the discharge chamber at the level of $10^{-2}-10^{-3}$ Torr). These experiments are planned in the nearest future.

\section{Acknowledgements}

The work was supported by the Russian Science Foundation, project No. 14-12-00609.

\section{References}

1. V.L. Bratman, I.V. Izotov, Yu.K. Kalynov, V.A. Koldanov, A.G. Litvak, S.V. Razin, A.V. Sidorov, V.A. Skalyga, and V.G. Zorin. Features of the plasma glow in the low pressure terahertz gas discharge. // Phys. Plasmas. 2013. V. 20. P. 123512.

2. M.Yu. Glyavin, S.V. Golubev, V.G. Zorin, I.V. Izotov, A.G. Litvak, A.G. Luchinin, M.V. Morozkin, S.V. Razin, A.V. Sidorov, and V.A. Skalyga. The discharge maintained by high-power terahertz radiation in a nonuniform gas flow. // Radiophys. Quantum Electronics. 2014. V. 56. No 8-9. P. $561-565$.

3. M.Yu. Glyavin, S.V. Golubev, I.V. Izotov, A.G. Litvak, A.G. Luchinin, S.V. Razin, A.V. Sidorov, V.A. Skalyga, and A.V. Vodopyanov. A point-like source of extreme ultraviolet radiation based on a discharge in a nonuniform gas flow, sustained by powerful gyrotron radiation of terahertz frequency band. // Appl. Phys. Letters. 2014. V. 105. P. 174101

4. S.V. Razin, A.V. Sidorov, S.V. Golubev, A.V. Vodopyanov, M.Yu. Glyavin, I.V. Izotov, A.G. Litvak, A.G. Luchinin, M.V. Morozkin, V.A. Skalyga. // Proceedings of the 9-th International workshop «Strong microwaves and terahertz waves: sources and applications». Nizhny Novgorod - Perm - Nizhny Novgorod, Russia, July 24-30. 2014. P.89-90.

5. A. V. Vodopyanov, M. Yu. Glyavin, S. V. Golubev, A. G. Luchinin, S. V. Razin, M. I. Safronova, A. V. Sidorov, and A. P. Fokin. Plasma Density in Discharge Sustained in Inhomogeneous Gas Flow by High-Power Radiation in the Terahertz Frequency Range. // Technical Physics Letters. 2017. V. 43. No. 2. P. 186-189.

6. http://www.nist.gov/pml/data/asd.cfm 